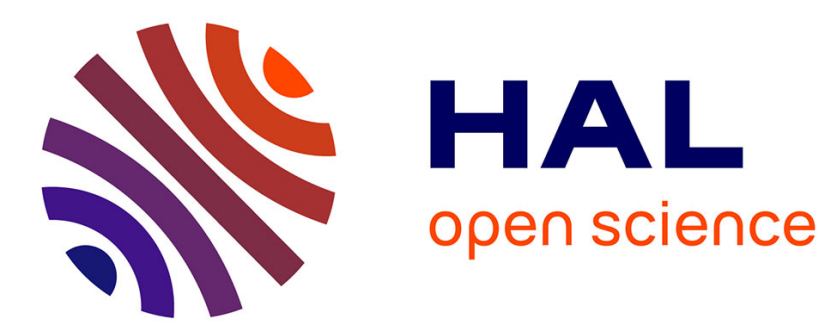

\title{
A Landau-de Gennes theory of nematic polymers
}

\author{
A. ten Bosch, P. Maïssa, P. Sixou
}

\section{To cite this version:}

A. ten Bosch, P. Maïssa, P. Sixou. A Landau-de Gennes theory of nematic polymers. Journal de Physique Lettres, 1983, 44 (3), pp.105-111. 10.1051/jphyslet:01983004403010500 . jpa-00232149

\section{HAL Id: jpa-00232149 https://hal.science/jpa-00232149}

Submitted on 1 Jan 1983

HAL is a multi-disciplinary open access archive for the deposit and dissemination of scientific research documents, whether they are published or not. The documents may come from teaching and research institutions in France or abroad, or from public or private research centers.
L'archive ouverte pluridisciplinaire HAL, est destinée au dépôt et à la diffusion de documents scientifiques de niveau recherche, publiés ou non, émanant des établissements d'enseignement et de recherche français ou étrangers, des laboratoires publics ou privés. 
Classification

Physics Abstracts

$61.30 \mathrm{C}-36.20 \mathrm{E}$

\title{
A Landau-de Gennes theory of nematic polymers
}

\author{
A. ten Bosch, P. Maïssa and P. Sixou \\ Laboratoire de Physique de la Matière Condensée (*), 28, av. Valrose, 06034 Nice Cedex, France
}

(Reçu le 26 août 1982, révisé le 25 octobre, accepté le 13 décembre 1982)

\begin{abstract}
Résumé. - On exprime l'énergie libre d'un polymère nématique semi-flexible sous forme d'un développement de Landau-de Gennes. Les paramètres qui interviennent sont le degré de polymérisation, l'élasticité propre de la chaîne et l'interaction quadrupolaire moyenne entre monomères. On étudie l'influence de la longueur et de la flexibilité des chaînes sur la température, le paramètre d'ordre et la chaleur latente de la transition nématique-isotrope.

Abstract. - For a semi-flexible, nematic polymer, the Landau-de Gennes expansion for the free energy of the homogeneous melt is derived. The theory contains three parameters : the degree of polymerization, the inherent elasticity of the polymer chain, and the quadrupolar mean field between monomers. The dependence on chain length and chain flexibility of the transition temperature, the order parameter and the latent heat of the nematic-isotropic transition is discussed.
\end{abstract}

1. Introduction. - A mean field theory has been developed to calculate phase diagrams and order parameters in flexible nematic polymers and polymer mixtures [1] and in lipid bilayers [2,3]. The addition of a bending elasticity of the polymer chain to the aligning, orientation dependent Van der Waals interaction leads to new effects, differing from the well-known results of MaierSaupe theory for rigid rod small molecule liquid crystals.

In the conventional liquid crystal systems, Landau-de Gennes theory [4] has proven to be as good as mean field theory in providing a semi-quantitative description of the nematic-isotropic transition. Furthermore, the Landau-de Gennes expansion is easier to handle and, by including spatial variations of the order parameter, applicable also to fluctuation phenomena. It is less satisfactory in the number of unknown phenomenological parameters it contains. By comparison to the expansion of the mean field free energy in powers of the order parameter, these coefficients can be identified with the parameters of the mean field theory.

This method has been adopted in lipid bilayers [3] and will be applied here to nematic polymers in the melt and used to calculate thermodynamic properties.

In the vicinity of the nematic-isotropic phase transition, the free energy of a polymer chain is written as an expansion in the order parameter $S$ :

$$
F(S, T)=F_{0}(T)+A\left(T-T^{*}\right) S^{2}-B S^{3}+C S^{4},
$$

(*) L.A. 190 au C.N.R.S. 
$F_{0}(T)$ is the free energy of the isotropic phase. The spatially invariant order parameter is given by the thermodynamic equilibrium value which minimizes the free energy :

$$
\frac{\partial F}{\partial S}(S, T)=0
$$

From(1), we obtain a first order phase transition at a critical temperature $T_{\mathrm{c}}$ :

$$
\begin{aligned}
& T>T_{\mathrm{c}}: S=0, \\
& T<T_{\mathrm{c}}: S=\frac{3}{8} \frac{B}{C}\left(1+\sqrt{1-\frac{32 A C\left(T-T^{*}\right)}{9 B^{2}}}\right),
\end{aligned}
$$

where

$$
T_{\mathrm{c}}=T^{*}+\frac{B^{2}}{4 A C}
$$

and the critical order parameter $S_{\mathrm{c}}$ at $T_{\mathrm{c}}$ is

$$
S_{\mathrm{c}}=\frac{B}{2 C}
$$

From (3), we can also calculate the temperature coefficient of the order parameter at the critical temperature $\mathrm{d} S / \mathrm{d} T\left(T=T_{\mathrm{c}}\right)$. This allows a study of the rate of increase of the orientational order near $T_{\mathrm{c}}$. A further quantity of interest is the latent heat of transition per polymer $\Delta h_{\mathrm{c}}$. Since at $T_{\mathrm{c}}$, $F\left(T_{\mathrm{c}}\right)=F_{0}\left(T_{\mathrm{c}}\right)$, therefore $\Delta h_{\mathrm{c}}$ is given by the difference in entropy between nematic $\left(s_{\mathrm{c}}\right)$ and isotropic $\left(s_{0}\right)$ phases : $\Delta h_{\mathrm{c}}=T_{\mathrm{c}}\left(s_{\mathrm{c}}-s_{0}\right)$. If the temperature dependence of the coefficients $A, B$ and $C$ is known, then $s=-\frac{\partial F}{\partial T}(S, T)$ can be calculated from (1).

To obtain expressions for these coefficients for a nematic polymer, we use the elastic worm-like chain model $[5,6]$. In contrast to small molecule, rigid rod liquid crystals, polymer liquid crystal chains have an inherent flexibility which effects the ordering in the nematic phase. To take this into account, the polymer chain is described by a continuous elastic line of given bending cons$\tan t \kappa$. If $\mathbf{r}(s)$ is the direction of the tangent to the line at contour length $s$ from the origin, the chain elastic energy is $\frac{1}{2} \int_{0}^{L} \mathrm{~d} s \kappa\left(\frac{\partial \mathbf{r}}{\partial s}\right)^{L}$. All lengths are measured in units of monomer length, so that $L$ denotes the degree of polymerization of the chain.

In addition, attractive (Van der Waals) and repulsive (steric) intermolecular forces of quadrupolar symmetry must be included. In the mean field approximation, the orientation dependent part of the potential on a polymer chain is written as $\int_{0}^{L} \mathrm{~d} s v(s)$. Here, $v(s)$ is the effective potential on a single segment at contour length $s$. If $\theta(s)$ is the angle of the tangent $\mathbf{r}(s)$, then

$$
v(s)=-\bar{v} S\left(\frac{3}{2} \cos ^{2} \theta(s)-\frac{1}{2}\right)
$$

The quantity $L S$ is the average orientation of a polymer chain, $S$ being the average orientation per monomer for a number $L$ of monomers

$$
S=\frac{1}{L}\left\langle\int_{0}^{L} \mathrm{~d} s\left(\frac{3}{2} \cos ^{2} \theta(s)-\frac{1}{2}\right)\right\rangle .
$$


In polymer systems, the spatial average interaction energy $\bar{v}$ can depend on chain conformation and chain length. For sufficiently short, semi-rigid chains, each monomer sees the bare field resulting from $L$ monomers on an adjacent chain. Therefore, for $L / q \rightarrow 0$,

$$
\bar{v}=v_{0} L \quad \text { and } \quad v(s)=-v_{0} L S\left(\frac{3}{2} \cos ^{2} \theta(s)-\frac{1}{2}\right) .
$$

The interaction between monomers, $v_{0}$, is a constant, as in rigid rod systems [7-9]. As the chain length increases, the interchain potential in the melt is screened by the presence of the other chains [10], and in the limit $q / L \rightarrow \infty$, the potential on a chain segment $\bar{v}=v_{x}$ becomes independent of chain length [11]. Therefore, for $q / L \rightarrow 0$

$$
v(s)=-v_{\infty} S\left[\frac{3}{2} \cos ^{2} \theta(s)-\frac{1}{2}\right] .
$$

This limit has been used in long chain polymer systems [2, 12].

In the intermediate range, the chain length dependence of $\bar{v}$ requires a self-consistent calculation of the spatial and orientational statistical distribution of the polymers.

In the intermediate range, the chain length dependence of the effective chain-monomer interaction may be more complex. In the following, we will consider the limiting cases which concern short and long semi-flexible chains only.

Fluctuations of the local nematic axis by the flexible polymer can lead to renormalization of the effective interaction parameter [6]. In nematic polymer melts, quenching of splay deformations is expected and these effects are neglected, as well as spatial variation of the order parameter [8].

2. Landau-de Gennes expansion. - The free energy of an elastic chain including orientation dependent Van der Waals interactions can be calculated from the functional integral of the partition function $F=-K T \ln Z$ where $\beta=1 / K T$ and

$$
Z=\int \mathrm{d}\{\operatorname{conf}\} \exp \left(-\beta \int_{0}^{L} \mathrm{~d} s\left[\frac{\kappa}{2}\left(\frac{\partial \mathbf{r}}{\partial s}\right)^{2}+v(s)-\frac{1}{2}\langle v(s)\rangle\right]\right) .
$$

As in small molecule liquid crystals, the exponential in the nematic potential $v(s)$ is expanded in powers of the order parameter :

$$
\exp \left(-\beta \int_{0}^{L} \mathrm{~d} s v(s)\right)=1+\beta \int_{0}^{L} \mathrm{~d} s v(s)+\cdots .
$$

Making use of the relation

$$
\ln Z=\ln Z_{0}+\frac{Z-Z_{0}}{Z_{0}}-\frac{1}{2}\left(\frac{Z-Z_{0}}{Z_{0}}\right)^{2}
$$

where $Z_{0}=4 \pi$ is the partition function of the isolated elastic chain, we find the coefficients $A, B$, $C$ and $T^{*}$ in (1).

Then $A=\bar{v} L / 2 T$ and the remainder of the coefficients are given by averages on the elastic chains; for example

$$
\begin{aligned}
K T^{*}=\frac{\bar{v}}{L} \int \frac{\mathrm{d}\{\operatorname{conf}\}}{Z_{0}} \int_{0}^{L} \mathrm{~d} s^{\prime} \mathrm{d} s^{\prime \prime}\left(\frac{3}{2} \cos ^{2} \theta\left(s^{\prime}\right)-\frac{1}{2}\right)\left(\frac{3}{2} \cos ^{2} \theta\left(s^{\prime \prime}\right)-\frac{1}{2}\right) \times & \\
& \times \exp \left(-\frac{\beta \kappa}{2} \int_{0}^{L}\left(\frac{\partial \mathbf{r}}{\partial s}\right)^{2} \mathrm{~d} s\right) .
\end{aligned}
$$


The functional integrals can be solved by introducing the statistical weight $G_{0}\left(\mathbf{R} \mathbf{R}^{\prime} L\right)$ on the elastic chain $[11,13]$

$$
G_{0}\left(\mathbf{R}, \mathbf{R}^{\prime}, L\right)=\int_{\mathbf{r}(0)=\mathbf{R}}^{\mathbf{r}(L)=\mathbf{R}^{\prime}} \mathrm{d}\{\operatorname{conf}\} \exp \left(-\frac{\beta \kappa}{2} \int_{0}^{L}\left(\frac{\partial \mathbf{r}}{\partial s}\right)^{2} \mathrm{~d} s\right) .
$$

The function $G_{0}\left(\mathbf{R} \mathbf{R}^{\prime} L\right)$ describes the probability for a certain chain configuration given by the initial tangent direction $(\mathbf{R}=\mathbf{r}(0))$ and the final tangent direction $\left(\mathbf{R}^{\prime}=\mathbf{r}(L)\right)$. The spherical symmetry of $G_{0}$ suggests expansion in spherical harmonics [5] $Y_{n}(\mathbf{R})$

$$
G_{0}\left(\mathbf{R}, \mathbf{R}^{\prime}, L\right)=\sum_{n} Y_{n}(\mathbf{R}) Y_{n}\left(\mathbf{R}^{\prime}\right) \mathrm{e}^{-E_{n} L},
$$

and the eigenvalues are found to be

$$
E_{n}=\frac{n(n+1)}{2 \beta \kappa}
$$

Then for example

$$
\begin{gathered}
K T^{*}=\frac{1}{5}\left(\frac{2 L}{E_{2}}-\frac{2}{E_{2}^{2}}\left(1-\mathrm{e}^{-E_{2} L}\right)\right) \frac{\bar{v}}{L}, \\
B=\frac{1}{105} \frac{1}{E_{2}^{2}}\left(\left(2+\mathrm{e}^{-E_{2} L}\right) 2 L-\frac{8}{E_{2}}\left(1-\mathrm{e}^{-E_{2} L}\right)+\frac{1}{E_{2}}\left(1-\mathrm{e}^{-2 E_{2} L}\right)\right) \frac{(\bar{v})^{3}}{(K T)^{2}},
\end{gathered}
$$

and $C$ is a similar (but complicated) expression in $L$ and $E_{2}$.

Note that the rigid rod system is a singular solution of (14) for which $\kappa \rightarrow \infty$ and $G_{0}=\delta\left(\mathbf{R}-\mathbf{R}^{\prime}\right)$. The coefficients of the Maier-Saune theory are obtained in this case : $K T^{*}=\frac{\bar{v}}{5} L, A=\frac{\bar{v} L}{2 T}$, $B=\frac{1}{105} \frac{(\bar{v} L)^{3}}{(K T)^{2}} ; C=\frac{1}{700} \frac{(\bar{v} L)^{4}}{(K T)^{3}}$.

3. Phase transition of nematic polymers - We give results for the nematic-isotropic phase transition and discuss chain length dependence for a fixed value of chain elasticity $\kappa$. The bending elastic constant is related to the free chain persistence length $q=\beta \kappa$, as shown by the calculation of the average end-to-end vector in [5].

For short chains $L / q \rightarrow 0$, and neglecting the temperature dependence of $q$, we find :

$$
\begin{aligned}
& A=\frac{v_{0} L^{2}}{2 T} \\
& B=\frac{\left(v_{0} L^{2}\right)^{3}}{105(K T)^{2}}(1-L / q), \\
& C=\frac{2\left(v_{0} L^{2}\right)^{4}}{735(K T)^{3}}(q / 3 L)^{3}
\end{aligned}
$$

The supercooling temperature

$$
K T^{*}=\frac{v_{0} L^{2}}{5}(1-L / q)
$$


and the nematic-isotropic transition temperature

$$
T_{\mathrm{c}}=T^{*}\left(1+\frac{9}{4}\left(\frac{L}{q}\right)^{3}\right),
$$

vary quadratically with the chain length and are independent of chain flexibility to order $L / q$. The critical order parameter $S_{\mathrm{c}}=\frac{7}{20}\left(\frac{3 L}{q}\right)^{3}$ increases with chain length in this regime, but the negative temperature coefficient at the transition(3) of the order parameter $S(T)$ decreases and the curves flatten. Finally the latent heat of transition per polymer chain $\Delta h_{\mathrm{c}} \cong \frac{v_{0} L^{2}}{2} S_{\mathrm{c}}^{2}$ increases rapidly.

In the other limit of long, semi-flexible chains $q / L \rightarrow 0$ and we obtain :

$$
\begin{aligned}
A & =\frac{v_{\infty} L}{2 T}, \\
B & =\frac{4}{945}\left(\frac{q}{L}\right)^{2} \frac{\left(v_{\infty} L\right)^{3}}{(K T)^{2}} \\
C & =\frac{1}{675}\left(\frac{q}{L}\right)^{2} \frac{\left(v_{\infty} L\right)^{4}}{(K T)^{3}}
\end{aligned}
$$

These coefficients give the following results for the thermodynamic properties near the phase transition. The supercooling temperature

$$
K T^{*}=\frac{2}{15} v_{\infty} q(1-q / 3 L),
$$

and the critical temperature

$$
T_{\mathrm{c}}=T^{*}\left(1+\frac{20}{441} q / L\right),
$$

are independent of chain length to order $q / L$ for long, flexible chains. The critical order parameter is small $S_{\mathrm{c}}=\frac{4}{21} q / L$ and the transition appears almost second order. For the temperature dependence of the order parameter near $T_{\mathfrak{c}}$, we find a saturation to a constant value at long chain lengths : $\frac{\partial S^{\left(T=T_{\mathrm{c}}\right)}}{\partial T} \cong-\frac{21}{5 T_{\mathrm{c}}}$.

Finally, again neglecting temperature dependence of the chain persistence length, we calculate the latent heat of transition per chain $\Delta h_{\mathrm{c}}=\frac{v_{\infty} L}{2} S_{\mathrm{c}}^{2}=\frac{8}{441}\left(v_{\infty} q\right) q / L$.

We find in this regime a decrease of latent heat with degree of polymerization.

4. Discussion. - It would also be possible, as in conventional liquid crystals, to determine the expansion coefficients $A, B, C$ and $T^{*}$ from experimental results. Information on the parameters of the mean field theory could thereby be obtained.

For comparison, the rigid rod model gives similar behaviour for the molecular weight dependence of the expansion coefficients and of the critical properties in the low molecular weight 
region - deviations occur in the high molecular weight region and as the presence of chain flexibility becomes apparent.

The statistical theory of wormlike nematic polymers presented in [14] is in qualitative agreement with our results in the high molecular weight limit although the method is quite different and a direct comparison difficult.

By addition of the appropriate free energy expression, the Landau-de Gennes theory for mixtures with nematic polymers is also obtained. For compatible mixtures with a small molecule liquid crystal, the phase diagrams are in agreement with the mean field calculations [1]. The quantitative behaviour of the order parameter is also found, although the expansion on the free elastic chain used in the Landau-de Gennes approach lowers the numerical values considerably. The effect of isotropic interactions (excluded volume), of less importance in the pure melt, can cause incompatibility for certain ranges of concentration in nematic polymer mixtures, as found in ordinary, flexible polymers.

In a mixture of a liquid crystal and a flexible, non-nematic polymer, a combined Flory, MaierSaupe theory gives a large biphasic interval near the nematic phases transition [15]. Local destruction of the liquid crystal order parameter by the polymer is demonstrated [16].

Experiments on thermotropic polymers are available [17-20] and from our model we are led to the following predictions for the critical properties at the isotropic-anisotropic phase transition on increasing the degree of polymerization of a polymer of given elasticity. At small values of chain length, the critical temperature, critical order parameter, slope of order parameter at $T_{\mathrm{c}}$ and latent heat of transition all increase with increasing chain length. For high molecular weights, the critical temperature, and the temperature coefficient of the order parameter saturate at maximum values determined by the range of the orientation dependent interaction. The critical order parameter and therefore the latent heat, diminish as the flexibility of the chain becomes more apparent. In the intermediate range of chain lengths, we therefore foresee a tendency to saturation on increasing the molecular weight for $T_{\mathrm{c}}$ and $\mathrm{d} S / \mathrm{d} T\left(T=T_{\mathrm{c}}\right)$. The critical order parameter and latent heat of transition first increase to a maximum value and then drop as the flexible polymer limit is approached.

In well fractionated samples of polyester melts $[17,18]$ and in a thermotropic cellulose derivative [19] the mesomorphic transition temperature has been measured as a function of molecular weight. A sharp increase is found in the low molecular weight samples, which then levels off. In high molecular weight samples, the transition temperature is independent of further increase in chain length. This is in agreement with our predictions. It would be of interest to examine the chain length dependence of other critical properties. It should be emphasized that only qualitative agreement with the scaling laws presented here can be expected. For example, temperature dependence of the intrinsic persistence length $q$ will alter the numerical values of the power laws.

It would also be possible to investigate the dependence on chain flexibility for fixed degree of polymerization with a similar behaviour of critical properties. The dilute solution persistence length can be obtained from intrinsic viscosity measurements and could, in principal, be used to deduce the value of the inherent elastic coefficient of the polymer chain. But the numerical values are uncertain and may be effected by solute-solvent interactions. Although fairly flexible in dilute solution, we expect the chains to stiffen in the nematic phase [1]. This would reduce entanglements and possible polymer deformations on application of external fields. A simple extension of the present theory to include electric, magnetic and elongational fields is then obtained by addition of the appropriate coupling interactions to the free energy [4]. An application to recent experiments on orientation of a mesogenic polymer in a static magnetic field [21] is envisioned.

Acknowledgments. - We would like to thank F. Brochard for clarifying discussions and R. Blumstein and D. Gray for the preprints of their work, and also F. Jähnig for the copy of his Habilitationsshrift. 
Note added in proof. - Further support is found in recent measurements of the heat of transition in a thermotropic polymer as a function of molecular weight (R. B. Blumstein, E. M. Stickles, A. Blumstein, Mol. Cryst. Liq. Cryst. 82 (1982) 205).

\section{References}

[1] Ten Bosch, A., Maïssa, P. and Sixou, P., Submitted for publication to J. Chem. Phys.

[2] JÄHNIG, F., J. Chem. Phys. 70 (1979) 3279.

[3] JÄHNIG, F., Mol. Cryst. Liq. Cryst. 66 (1979) 157.

[4] Sheng, P. and Priestly, E. B., Introduction to Liquid Crystals (Plenum Press).

[5] Saito, N., Takahashi, K. and Yunoki, Y., J. Phys. Soc. Jupun 22/1 (1967) 219.

[6] De Gennes, P. G., Proc. Polymer Liquid Crystals, Science und Technology, Santa Margherita (1981).

[7] Onsager, L., Ann. N.Y. Acud. Sci. 51 (1949) 627.

[8] DoI, M., J. Polym. Sci. 19 (1981) 229.

[9] Deblieck, R. and LeKKerkerker, H. N. W., J. Physique Lett. 41 (1980) L-351.

[10] Edwards, S. F., Proc. Phys. Soc. 88 (1966) 265.

[11] De Gennes, P. G., Sculing concepts in Polymer Physics (Cornell University Press) 1979.

[12] Flory, P. J. and RonCa, G., Mol. Cryst. Liq. Cryst. 54 (1979) 311.

[13] Freed, K. F., Adv. Chem. Phys. 22 (1972) 1.

[14] RoncA, G. and Yoon, D. Y., J. Chem. Phys. 76/6 (1982) 3295.

[15] Brochard, F., C.R. Hebd. Séan. Acad. Sci. 289 (1979) 229.

[16] Brochard, F., C.R. Hebd. Séan. Acad. Sci. 290 (1980) 485.

[17] Blumstein, A., Vilasagar, S., Ponrathnam, S., Clough, S. B., Maret, G. and Blumstein, R., J. Polym. Sci. 20 (1982) 877.

[18] BhADANI, S. N. and GRAY, D. G., Proceedings 28th IUPAC Macromolecular Symposium, Amherst 1982.

[19] Finkelmann, H., Luhmann, B., Rehage, G. and Stevens, H., To be published in Liquid crystuls und Ordered fluids, vol. 4.

[20] Fayolle, B., Noel, C. and Billard, J., J. Physique Colloq. 40 (1979) C3-485.

[21] Maret, G., Volino, F., Blumstein, R., Martins, A. F. and Blumstein, A., Proceedings 27th IUPAC Symposium on Macromolecules, Strasbourg 1981. 